\title{
Artifacts Removal of EEG Signals By the Application of ICA and Double Density DWT Algorithm
}

\author{
Vandana Roy ${ }^{1}$, Dr.(Mrs) Shailja Shukla ${ }^{2}$ \\ ${ }^{I}$ Research scholar (PhD), Department of Electronics \& Communication, Jabalpur Engineering College, \\ Jabalpur, Madhya Pradesh, India \\ 2. Professor \& Head of Department of Computer Science Engineering, Jabalpur Engineering College, Jabalpur, \\ Madhya Pradesh, India
}

\begin{abstract}
Independent Component Analysis is used for the automation and detection of brain artifacts. The Independent Component Analysis (ICA) here is used for the segmentation of artifact peaks in the signal. Then the Discrete Wavelet Transform is applied for multi-level transfer of signal data until the reception of significant result. We have extended our search and applied the Double Density Algorithm for the multi-level transfer. The results obtained were analyzed from the data set of EEG signals taken with a outsource reference. Since the method is parameter free implementations in clinical settings are imaginable.
\end{abstract}

Index Terms: Artifacts, EEG Signals, Double Density, Wavelet Denoising.

(C) 2014 Published by MECS Publisher. Selection and/or peer review under responsibility of the Research Association of Modern Education and Computer Science.

\section{Introduction}

EEG artifacts are the recorded signals that are non-cerebral in origin. The EEG signals are divided into one of two categories depending on their origin: physiological artifacts and non-physiological artifacts [1]. The amplitude of artifacts can be quite large relative to the size of amplitude of the cortical signals of interest. The most of common variety of artifacts include: power line artifact, eye movement, eye blinking, respiratory artifact, electrode popping, ECG artifact, sweat artifact, loose/broken electrode. The details of the effect of these categories are given in [2]. Artifacts are ubiquitous i.e. they are present in every EEG tracing. Artifacts on being obscure the EEG activity and render the EEG uninterruptable. Williams etal. 1985 said, "Artifacts can mimic almost any kind of cerebral activity and lead to serious interpretation."

EEG signals waves are complex, and has to be completed with its own set of perplexing nomenclature. The waves like radio stations are categorized by frequency of their emanations or by the nature of their shape. No,

* Corresponding author.

E-mail address: vandana.roy20@gmail.com 
wave is emitted alone but the conscious state of brain makes a wave more pronounced than other wave for an individual. Five types of waves are stated in [3].

Beta: The rate of change lies in the range of 13 to $30 \mathrm{~Hz}$. It is associated with active thinking, attention and focus. A high of $50 \mathrm{~Hz}$ can be seen in state of intense mental activities.

Alpha: The rate of change is in the range of 8 to $13 \mathrm{~Hz}$ with $30-50 \mu \mathrm{V}$ amplitude. The relaxed awareness and the in-attention states are considered to fall in this range. It is considered to be one of the most prominent ranges as beta with a maximum high of $20 \mathrm{~Hz}$ is suspected to be Alpha wave. A mindless or empty state of mind falls in alpha category easily distracted by opening of eyes or listening unfamiliar sounds.

Theta: Theta waves lies in 4 to $7 \mathrm{~Hz}$ range with an amplitude usually greater than $20 \mu \mathrm{V}$. Emotional stress specially frustration or disappointment state of brain produces this category of signals. A deep meditation is state of this category. These waves see a max high of $7 \mathrm{~Hz}$.

Delta: with a variable amplitude and range of 0.5 to $4 \mathrm{~Hz}$ signals falls in this category. Deep sleeping state is the corresponding body activity of this state. However this state experiences sound artifact signals produced by the movement of large neck muscles and jaw movement with genuine delta responses. The original signals are produced in the deep brain and are severely attenuated in their path towards skull.

Gamma: Gamma waves lies in the range more than $35 \mathrm{~Hz}$. This state is considered to reflect the mechanism of consciousness.

Many methods have been proposed to remove eye movement and blink artifacts from EEG recordings. As in [4] some of them include simple rejection of contaminated EEG epochs which results in a considerable loss of collected information. Often regression in the time or frequency domain is performed on simultaneous EEG and electrooculography (EOG) recordings to derive parameters characterizing the appearance and spread of EOG artifacts in the EEG channels. However, EOG records also contain brain signals, so regressing out EOG activity inevitably involves subtracting a portion of the relevant EEG signal from each recording as well. Since many noise sources, include muscle noise, electrode noise and line noise, have no clear reference channels, regression methods cannot be used to removed them.

Hence a new and approved method of Independent Component Analysis (ICA) is applied for the detection and removal of artifacts. We have used the Wavelet Denoising techniques along with the ICA for the removal of artifacts from the signals. By ICA we will calculate the mean and variance of the signals and subtract it from base signal to distribute the signal in high and low frequency components. DWT will is then applied to separate both the signals and get the expected outcome.

\section{Literature Survey}

State of the Art method was introduced initially to eradicate the artifacts from the base signal. The method was made capable to sense the EEG and remove the artifacts simultaneously. The EOG electrodes are placed close to EEG electrodes; they contain a certain amount of brain activity. Thus removing the artifacts cause distortion in EEG signals. Hence further complete methods introduced in this field are summarized in this segment.

Authors in [5] have compared three ICA algorithms i.e. Infomax, SOBI and Fast ICA. These methods allow high degree sensitive automated detection of small non-brain artifacts than the directly applied methods to the scalp channel data. The authors tested all five methods for the performance analysis to detect various types of artifacts by separately optimizing and then applying them to artifact free EEG signals in which manually artifacts of various kinds were added. Except for the muscle artifact detection all the methods involving ICAdecomposed data were superior to raw scalp data.

[6] Presents a method for the removal of ocular artifacts in Electro-Encephalogram (EEG) records. The noise source creates the difficulty in the analysis of EEG and clinical information. The authors have proposed the method of Removal of Ocular Artifacts in the EEG through Wavelet Transform without the use of an EOG Reference Channel. The data under consideration is outsourced from a website and sampled at a rate of 128 
samples per second. The data is passed through for the identification of spikes and ocular artifacts spike zone. They finally proved that the method provides easy and less complex results with wavelet denoising.

In [7] authors proposed a method for the removal of artifacts from High Density EEG recording during walking and running. They recorded a channel-based artifact template regression procedure and a subsequent spatial filtering approach to remove gait-related movement artifact from EEG signals. First a stride time wrapping for removal of gait-artifact during visual oddball discrimination task and then ICA (isomax algorithm) is applied to parse the channel-based low noise EEG signals into maximally ICs followed by a componentbased template regression. This method reduced spectral power in 1.5 to $8.5 \mathrm{~Hz}$ frequency range in condition of walking and running. In walking conditions, gait-related artifact was insubstantial: event-related potentials (ERPs), which were nearly identical to visual oddball discrimination events while standing, were visible before and after applying noise reduction. In the running condition, gait-related artifact severely compromised the EEG signals: stable average ERP time-courses of IC processes were only detectable after artifact removal. These findings show that high density EEG can be used to study brain dynamics during whole body movements and that mechanical artifact from rhythmic gait events may be minimized using a template regression procedure.

\section{Proposed Methodology}

In this paper we have used the Independent Component Analysis (ICA) for the segmentation of artifact peaks in the signal. Then the Double Density Wavelet Transform is applied for multi-level transfer of signal data until the reception of significant result.

\subsection{Independent Component Analysis}

Consider the two signals $\mathrm{x}_{1}(\mathrm{t})$ and $\mathrm{x}_{2}(\mathrm{t})$. The $\mathrm{x}$ is amplitude of the signals and $\mathrm{t}$ is the time frame involved. Let the output signal is $s_{1}(t)$ and $s_{2}(t)$, the function is defined by the equation:

$$
\begin{aligned}
& \mathrm{x}_{1}(\mathrm{t})=\mathrm{a}_{11} \mathrm{~s}_{1}+\mathrm{a}_{12} \mathrm{~s}_{2} \\
& \mathrm{x}_{2}(\mathrm{t})=\mathrm{a}_{21} \mathrm{~s}_{1}+\mathrm{a}_{22} \mathrm{~s}_{2}
\end{aligned}
$$

Where, $a_{11} a_{12} a_{21} a_{22}$ are some parameters defining the feasibility of the equation. One can determine the values of $\mathrm{s} 1(\mathrm{t})$ and $\mathrm{s} 2(\mathrm{t})$ by only the values of $\mathrm{x}_{1}(\mathrm{t})$ and $\mathrm{x}_{2}(\mathrm{t})$. This is called as the cocktail party problem. Human mind is able to process and analyze the required sound in the noise. In communication channel the signal is recognized using ICA technique. e. The recently developed technique of Independent Component Analysis (ICA), can be used to estimate the $\mathrm{a}_{\mathrm{ij}}$ based on the information of their independence, which allows us to separate the two original source signals $\mathrm{s}_{1}(\mathrm{t})$ and $\mathrm{s}_{2}(\mathrm{t})$ from their mixtures $\mathrm{x}_{1}(\mathrm{t})$ and $\mathrm{x}_{2}(\mathrm{t})$.

ICA Preprocessing: To perform the operations of ICA it is necessary to pre-process the data. Here we discuss the necessary factors involved in this.

Centering: It is the most basic and mandatory step in pre-processing of ICA. The method centers $\mathbf{x}$ i.e. subtraction of mean vector $\mathrm{m}=\mathrm{E}\{\mathbf{x}\}$ so as to make $\mathrm{x}$ a zero-mean variable. Similarly we consider $\mathrm{s}$ is also zero-mean. After estimating the mixing matrix $\mathbf{A}$ with centered data, we can complete the estimation by adding the mean vector of $\mathbf{s}$ back to the centered estimates of $\mathbf{s}$. The mean vector of $\mathbf{s}$ is given by $\mathbf{A}^{-1} \mathbf{m}$, where $\mathbf{m}$ is the mean that was subtracted in the preprocessing.

Whitening: The second frequent preprocessing step in ICA is de-correlating (and possibly dimensionality reducing), called whitening. In whitening the sensor signal vector $\mathrm{x}$ is transformed using formula

$$
y=W \mathrm{x} \text {, so } E\left\{y y^{T}\right\}=I_{l} \text {, }
$$


Wherey $\in R^{l}$, is the $l-$ dimensional $(l \cdot n)$ whitened vector, and $\mathrm{W}$ is $l \times n$ whitening matrix. The purpose of whitening is to transform the observed vectorx linearly so that we obtain a new vector $\mathbf{y}$ (which is white) which elements are uncorrelated and their variances are equal to unity. Whitening allows also dimensionality reduction, by projecting of $\mathbf{x}$ onto first $l$ eigenvectors of the covariancematrix of $\mathbf{x}$.

Whitening is usually realized using the Eigen-value decomposition (EVD) of the covariance matrix $E\left\{y y^{T}\right\} \in R^{n \times N}$ of observed vector $\mathbf{x}$

$$
R_{x x}=E\left\{x x^{T}\right\}=E_{x} \Lambda_{x}^{1 / 2} \Lambda_{x}^{1 / 2} E_{x}^{T}
$$

Here, $E \mathbf{x} \in R^{n \times n}$ is the orthogonal matrix of eigenvectors of $R_{\mathrm{xx}}=E\left\{\mathbf{x x}^{T}\right\}$ and $\Lambda$ is the diagonal matrix of its eigenvalues

$$
\Lambda_{x}=\operatorname{diag}\left(\lambda_{1}, \lambda_{2}, \ldots, \lambda_{n}\right)
$$

With positive Eigen values $\lambda_{1} \geq \lambda_{2} \geq . . \geq \lambda_{n} \geq 0$. The whitening matrixes can becomputed as

$$
W=\Lambda_{x}^{-1 / 2} E_{x}^{T}
$$

And consequently the whitening operation can be realized using formula

$$
y=\Lambda_{x}^{-1 / 2} E_{x}^{T} x=W x
$$

Recalling that, $x=H s$, we can find from the above equation that

$$
y=\Lambda_{x}^{-1 / 2} E_{x}^{T} \quad H s=H_{\omega} s
$$

We can see that whitening transforms the original mixing matrix $\mathrm{H}$ into a new one, $H_{\omega}$

$$
H_{\omega}=\Lambda_{x}^{-1 / 2} E_{x}^{T} \quad H
$$

Whitening makes it possible to reduce the dimensionality of the whitened vector, by projecting observed vector into first $l(l \leq n)$ eigenvectors corresponding tofirst $l$ eigenvalues $\lambda_{1}, \lambda_{2}, \ldots, \lambda_{l}$, of the covariance matrix, $E_{\mathrm{x}}$. Then, the resulting dimension of the matrix $\mathrm{W}$ is, $l \times n$ and there is reduction of the size of observed transformed vector y from $n$ tol.

Output vector of whitening process can be considered as an input to ICA algorithm. The whitened observation vector $\mathrm{y}$ is an input to un-mixing (separation)operation

$$
s=A y
$$

Where, $\mathrm{A}$ is an original un-mixing matrix. An approximation (reconstruction) of the original observed vector $\mathrm{x}$ can be computed as,

$$
\tilde{x}=A s,
$$

Where $A=W_{\omega}^{-1}$. 
For the set of $N$ patterns $\mathrm{x}$ forming as columns the matrix $\mathrm{X}$ We can provide the following ICA model

$$
X=A S
$$

Where $S=\left[s_{1}, s_{2}, \cdots, s_{n}\right]$ is the $m \times N$ matrix which columns correspond toindependent component vectors $s_{i}=\left[s_{i 1}, s_{i 2}, \cdots, s_{i m}\right]^{T}$ discovered from the observation vector, $\mathrm{x}_{i}$. Consequently we can find the set $\mathrm{S}$ of corresponding independent component vectors as

$$
S=A^{-1} X \text {. }
$$

\section{Fast ICA Method:}

To understand this we use the ICA model given in [8]. A unit is considered to be something that represents processing element. Like for a neuron it has weight $\mathbf{W}$. The weight of several similar other elements considered as $\mathbf{w}_{\mathbf{1}}, \mathbf{w}_{\mathbf{2}}, \ldots . ., \mathbf{w}_{\mathbf{n}}$ should be determined. In outputs it must be done independent after each iteration in order to avoid convergence of same maxima. The best method is to do analysis one by one.

\section{Algorithms:}

i. Initialize $\boldsymbol{w}_{i}$

ii. Newton Phase

$$
w_{i}=E\left\{\tilde{x} g\left(w_{i}^{T} \tilde{x}\right)\right\}-E\left\{g^{\prime}\left(w_{i}^{T} \tilde{x}\right)\right\} w_{i}
$$

Where $\mathrm{g}$ is a function with one of the following form:

$$
\begin{aligned}
& g_{1}(y)=\tanh \left(a_{1} y\right), \\
& g_{2}(y)=y \exp \left(-\frac{1}{2} y^{2}\right), \\
& g_{3}(y)=4 y^{3}
\end{aligned}
$$

iii. Normalization

$$
W_{i}=\frac{1}{\left\|W_{i}\right\|} W_{i}
$$

iv. De-correlation

$$
w_{i}=w_{i}-\sum_{j=1}^{i-1} w_{i}^{T} w_{j} w_{j}
$$

v. Normalization like in step iii.

vi. Go to step ii. If not converged.

The statistical independence of unconstrained sources needs to be maximized. The divergences among spatially constrained source sensor projections are bounded to be decremented along with its supporting topographies. The Fast ICA algorithm here extracts the desired components that is a matrix (an array of artifact 
signals), computed by a corresponding matrix. This saves the critical time of computation, compared to the parallel ICs methods.

\subsection{B. Double Density DWT}

The wavelet technique came into existence to overcome the resolution limitations of spectral analysis of Fourier Transform and denoise the corrupted signal. Selesnick et al. [4] introduce the DDWT as the tight-frame equivalent of Daubechies orthonormal wavelet transform; the wavelet filters are of minimal length and satisfy certain important polynomial properties in an oversampled framework. Because the DDWT, at each scale, has twice as many wavelets as the DWT, it achieves slower shift sensitivity than the DWT. The algorithm upgraded in many forms from 1-D, 2-D up to double density complex [9]. The DDWT is implemented on discrete-time signals using the oversampled analysis and synthesis filter bank shown in Figure 4. The frequency responses for the analysis-bank filters are as shown in Figure 1, for a set of filters $h^{0}, h^{1}, h^{2}$ with lengths 7, 7, 5 respectively. The $h^{0}$ filter is a low pass filter, while the $h^{1}$ and $h^{2}$ filters are high pass filters with similar frequency magnitude responses. An iterated oversampled filter bank is created by the usual iteration on the low pass branches of the analysis and synthesis banks. Selesnick et al. show that the above DDWT implementation corresponds to a multi resolution analysis with a single scaling function $\emptyset$ and two wavelets $\varphi^{1}, \varphi^{2}$ as shown in Figure 1. Observe that the wavelets approximately satisfy the "half-delay" property $\varphi^{1}(t)=\varphi^{2}(t-1 / 2)$.

The double density DWT is applying the oversampled filter bank on a low-pass sub band signal c(n). In this paper we have used the double density algorithm along with the filter banks.
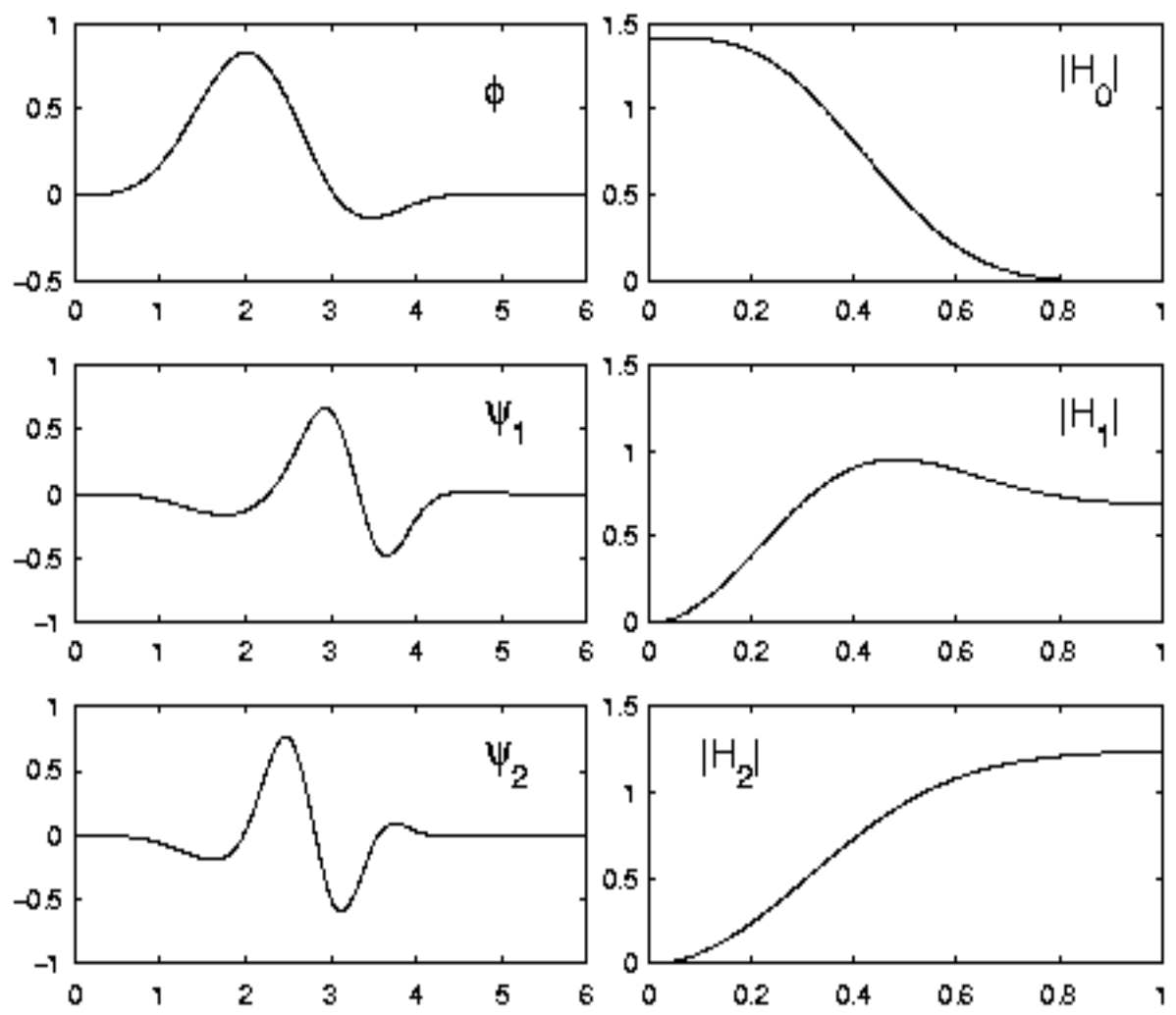

Fig. 1: Left column: basis functions, Right column: frequency responses. 


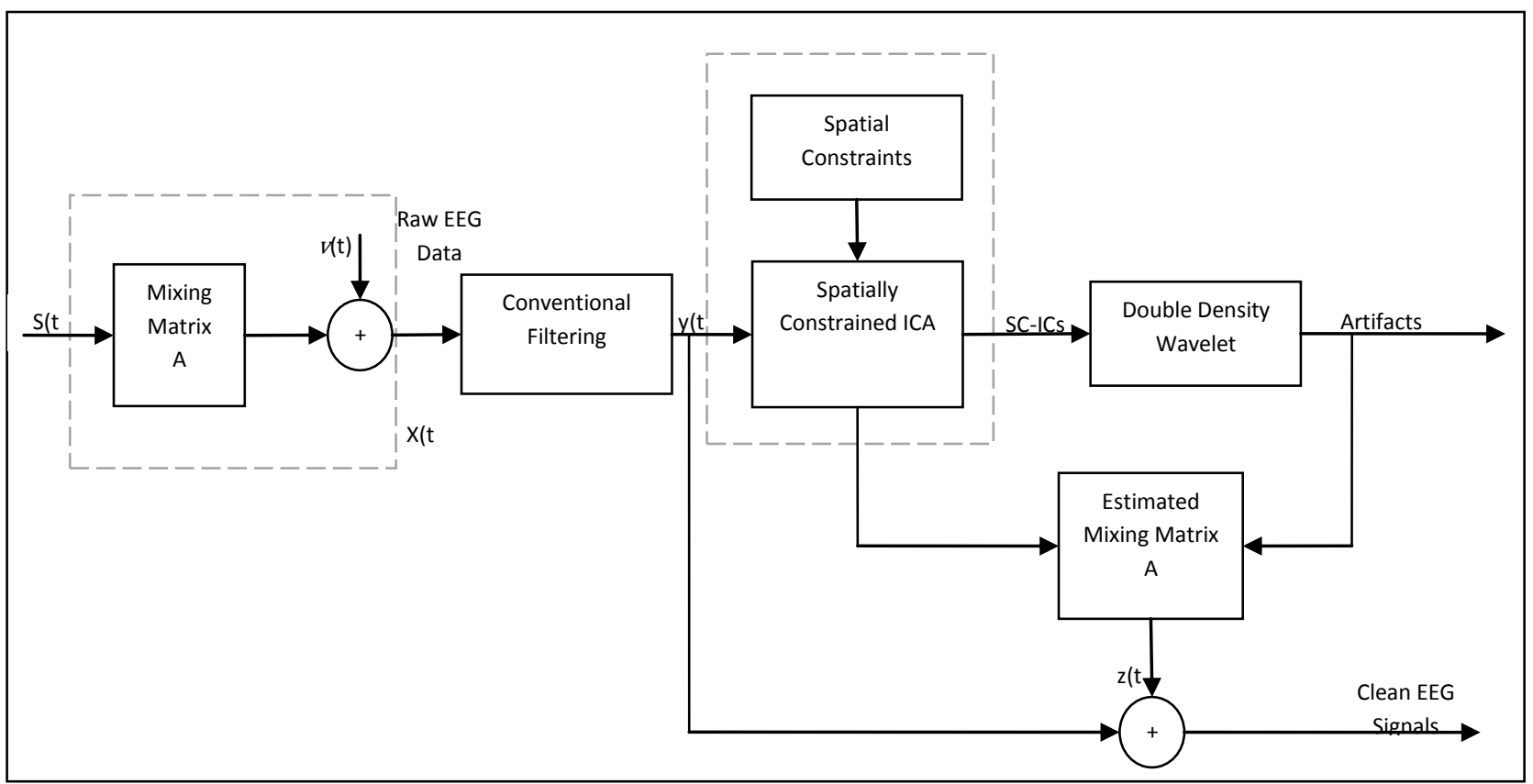

Fig.2: Proposed Flow Diagram of Overall Process of Artifact Removal

The reconstruction condition of filter bank of Figure 3 is derived as follows. We are using basic multi-rate identities we find $Y(z)$, the Z-transform of $y(n)$, in terms of $X(z)$ received as the output matrix of ICA [9].

$$
Y(z)=\left[\frac{1}{2} H_{0}(z) H_{0}\left(\frac{1}{z}\right)+H_{1}(z) H_{1}\left(\frac{1}{z}\right)\right] X(z)+\frac{1}{2} H_{0}(z) H_{0}\left(-\frac{1}{z}\right) X(-z)
$$

Where,

$\mathrm{H}_{0}$ and $\mathrm{H}_{1}$ are the filters.

For perfect reconstruction it is necessary that $\mathrm{Y}(\mathrm{z})=\mathrm{X}(\mathrm{z})$.

$$
\frac{1}{2} H_{0}(z) H_{0}\left(\frac{1}{z}\right)+H_{1}(z) H_{1}\left(\frac{1}{z}\right)=1
$$

$$
H_{0}(z) H_{0}\left(-\frac{1}{z}\right)=0
$$

This can be written as:

$$
H_{0}\left(e^{j w}\right) \overline{H_{0}\left(e^{j(w-\pi)}\right)}=0
$$



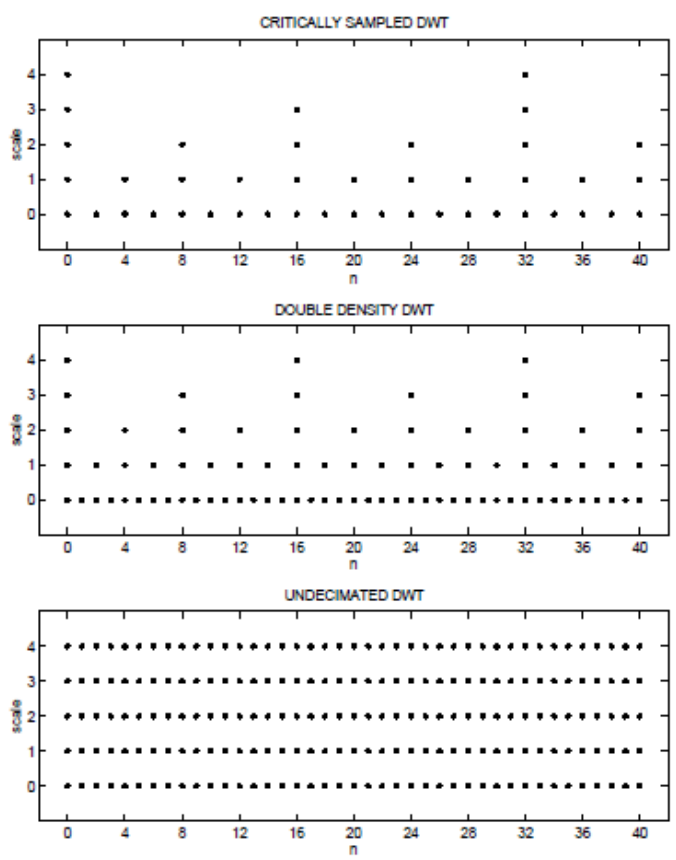

Fig. 3: Idealized time-frequency localization diagrams. The double density DWT gives a denser sampling of the time-frequency plane than the critically sample DWT. But unlike the un-decimated DWT, it maintains the same octave spacing.

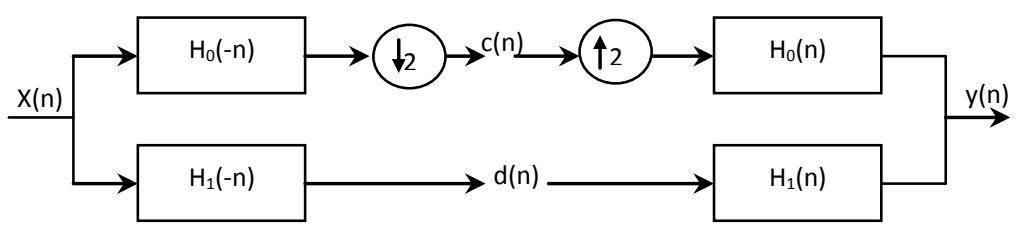

Fig. 4: An oversampled analysis and synthesis filter bank for which perfect reconstruction is impossible with realizable filters.

The $H_{0}\left(e^{j w}\right)$, is discrete Fourier transform of $H_{0}(n)$. The ideal low pass filter is given by:

$$
H_{0}\left(e^{j w}\right)=\left\{\begin{array}{l}
1|w|<\pi / 2 \\
0 \frac{\pi}{2}<|w|<\pi
\end{array}\right.
$$

satisfies the above equation.

\section{Dual-tree DWT}

The double-density dual-tree DWT proposed in this paper is based on concatenating two oversampled DWTs. The filter bank structure corresponding to the double-density dual-tree DWT consists of two oversampled iterated filter banks operating in parallel, similar to the dual-tree DWT. The oversampled filter bank is illustrated in Fig. 5. The iterated oversampled filter bank, corresponding to the implementation of the double- 
density dual-tree, is illustrated in Fig. 6. We will denote the filters in the first filter bank by $h_{i}(n)$ and the filters in the secondfilterbank by $g_{i}(n)$, for $i=0,1,2$. Note that in each of thefilterbanks to be considered in this paper, the synthesis filtersare the time-reversed versions of the analysis filters. The goal will be to design the six FIR filters so that they do the following [12].

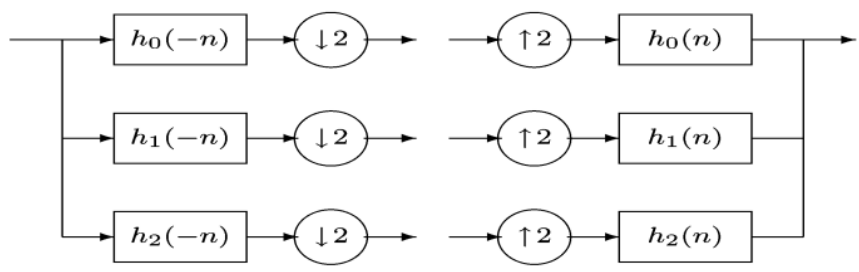

Fig. 5: Oversampled analysis and synthesis filter bank.

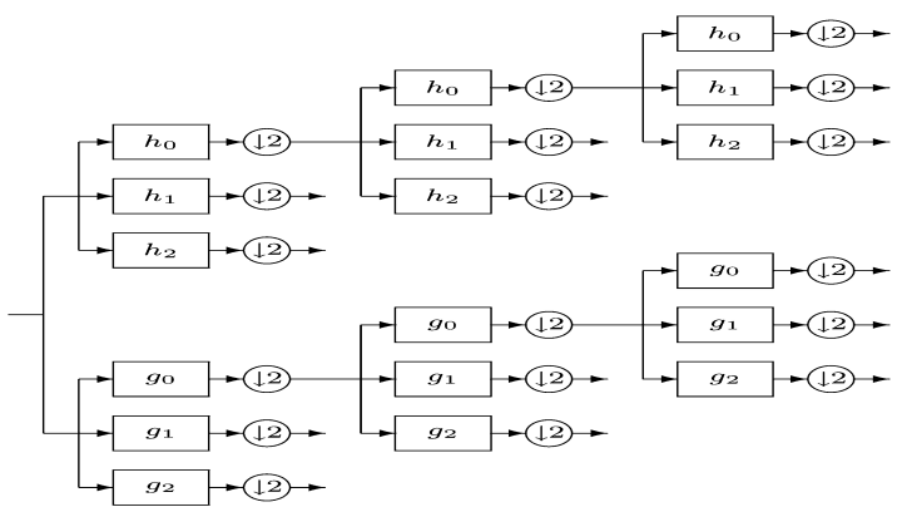

Fig. 6: Iterated filter bank for the double-density dual-tree DWT.

i. They satisfy the perfect reconstruction property.

ii. The wavelets form two (approximate) Hilbert transform pairs.

iii. The wavelets have specified vanishing moments.

iv. The filters are of short support.

The -transform of $h_{i}(n)$ is denoted by $H_{i}(z)$

$$
H_{i}(z)=Z T\left\{h_{i}(n)\right\}:=\sum_{n} h_{i}(n) Z^{-n}
$$

$G_{i}\left(e^{j \omega}\right)$ and is similarly defined. Throughout the paper, it is assumed that all filter coefficients $h_{i}(n), g_{i}(n)$ are real valued.The frequency response is given by

$$
H_{i}\left(e^{j \omega}\right)=\operatorname{DTFT}\left\{h_{i}(n)\right\}:=\sum_{n} h_{i}(n) e^{-j n \omega}
$$

And $G_{i}\left(e^{j \omega}\right)$ is similarly defined. The filters $h_{i}(n)$ and $g_{i}(n)$ should satisfy the perfect reconstruction (PR) conditions. From basic multi rate identities, the PR conditions are shown as below:

$$
\sum_{i=0}^{2} H_{i}(z) H_{i}(1 / z)=2
$$




$$
\sum_{i=0}^{2} H_{i}(z) H_{i}(-1 / z)=0
$$

And

$$
\begin{aligned}
& \sum_{i=0}^{2} G_{i}(z) G_{i}(1 / z)=2 \\
& \sum_{i=0}^{2} G_{i}(z) G_{i}(-1 / z)=0
\end{aligned}
$$

The scaling and wavelet functions are well-defined implicitly through the dilation and wavelet equations

$$
\begin{aligned}
& \emptyset_{n}(t)=\sqrt{2} \sum_{n} h_{o}(n) \emptyset_{h}(2 t-n) \\
& \psi_{h, 1}(t)=\sqrt{2} \sum_{n} h_{1}(n) \emptyset_{h}(2 t-n) \\
& \psi_{h, 2}(t)=\sqrt{2} \sum_{n} h_{2}(n) \emptyset_{h}(2 t-n)
\end{aligned}
$$

Where $\emptyset_{n}(t)$ and $\psi_{g, i}(t)$ are defined similarly. The Fourier transforms of the scaling functions and wavelets will be denoted as

$$
\begin{aligned}
& \Phi_{n}(\omega)=\mathcal{F}\left\{\emptyset_{n}(t)\right\}, \Phi_{g}(\omega)=\mathcal{F}\left\{\emptyset_{g}(t)\right\} \\
& \psi_{h, i}(\omega)=\mathcal{F}\left\{\psi_{h, i}(t)\right\}, \quad \psi_{g, i}(\omega)=\mathcal{F}\left\{\psi_{g, i}(t)\right\}
\end{aligned}
$$

The Hilbert transform of a function $f(t)$ will be denoted as $H\{f(t)\}$. Following the work by Kingsbury, we want the wavelets to form Hilbert transform pairs

$$
\begin{aligned}
& \psi_{g, 1}(t)=\mathcal{H}\left\{\psi_{h, 1}(t)\right\} \\
& \psi_{g, 2}(t)=\mathcal{H}\left\{\psi_{h, 2}(t)\right\}
\end{aligned}
$$

Recalling the definition of the Hilbert transform, can be described as:

$$
\Psi_{g, i}(\omega)=\left\{\begin{array}{c}
-j \Psi_{h, i}(\omega), \omega>0 \\
j \Psi_{h, i}(\omega), \omega<0
\end{array}\right.
$$

\section{Results and Discussion}

The proposed methodology was simulated on two data sets. First one was the EEG signals from rats. It is a 5 sec recording of two-channel EEG recording at the right and left frontal cortex of a male adult WAG/Rij rats. Signals were referenced to an electrode placed at the cerebellum and were filtered in the range of $1-100 \mathrm{~Hz}$ and digitized at $200 \mathrm{~Hz}$ [11]. Example A corresponds to a normal EEG and examples B, C, D and E contain spikewave discharges. The Discrete Wavelet Algorithm and Double Density Algorithm were applied and following results were oriented: 
Table 1: PSNR and MSE values on test samples using Discrete Wavelet Algorithm

\begin{tabular}{|c|c|c|}
\hline Samples & PSNR & MSE \\
\hline Raw_a & 37.3613 & 0.00038510 \\
\hline Raw_b & 41.6970 & 0.00038490 \\
\hline Raw_c & 36.1706 & 0.00038480 \\
\hline Raw_d & 38.3893 & 0.00038450 \\
\hline Raw_e & 35.4906 & 0.00038390 \\
\hline
\end{tabular}

Table 2: PSNR and MSE values on test samples using Double Density Algorithm

\begin{tabular}{|c|c|c|}
\hline Samples & PSNR & MSE \\
\hline Raw_a & 43.7700 & 0.00038510 \\
\hline Raw_b & 46.7562 & 0.00038500 \\
\hline Raw_c & 42.9276 & 0.00038460 \\
\hline Raw_d & 43.9147 & 0.00038410 \\
\hline Raw_e & 42.8613 & 0.00038390 \\
\hline
\end{tabular}

The second data set is Pattern Visual Evoked Potentials. Each data file is the recording on a different subject in the left occipital electrode (01), with linked earlobes reference. Each data file consists of a several artifactfree trials, with 512 data points (256 pre- and 256 post simulation) stored with sampling frequency of $250 \mathrm{~Hz}$. Data was pre-filtered in the range $0.1-70 \mathrm{~Hz}$. All trials correspond to target stimulation with an oddball paradigm [11].

Table 3: PSNR and MSE values on test samples using Discrete Wavelet Algorithm

\begin{tabular}{|c|c|c|}
\hline Samples & PSNR & MSE \\
\hline Infant_a & 9.8288 & 0.00038508 \\
\hline
\end{tabular}

Table 4: PSNR and MSE values on test samples using Double Density Algorithm

\begin{tabular}{|c|c|c|}
\hline Samples & PSNR & MSE \\
\hline Infant_a & 17.744 & 0.00038508 \\
\hline
\end{tabular}




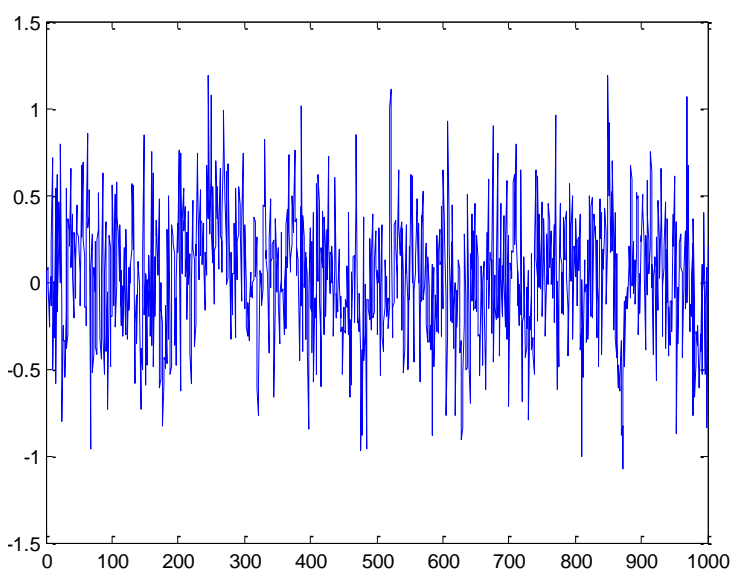

Fig. 7: Input EEG data signal for the test sample Raw_a

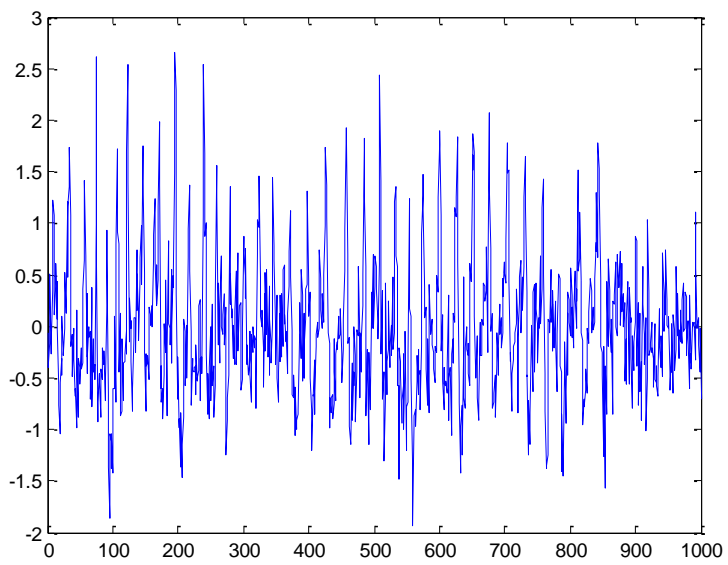

Fig. 8: Input EEG data signal for the test sample Raw_b

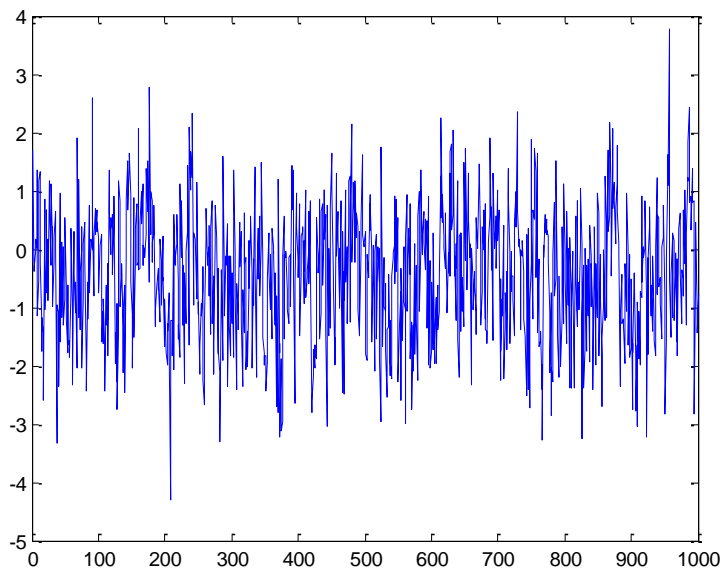

Fig. 9: Output EEG data signal for the test sample Raw_a 


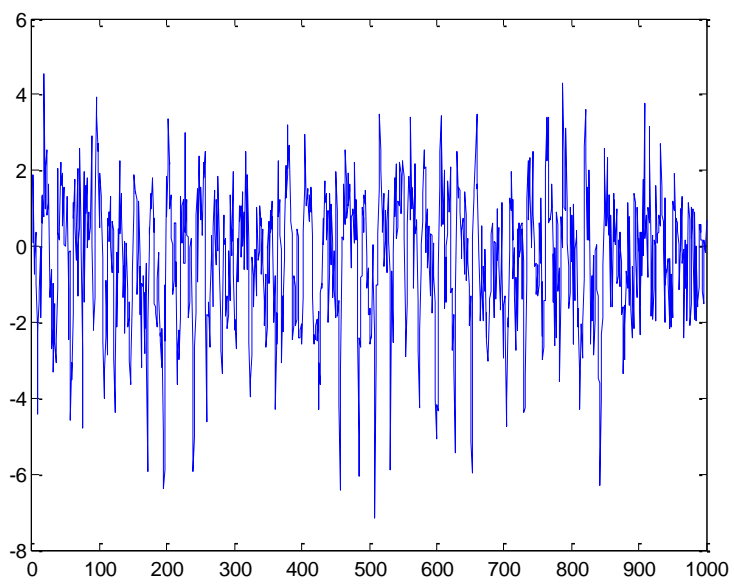

Fig. 10: Output EEG data signal for the test sample Raw_b

\section{Conclusion}

We studied the artifacts removal of brain signal reports in form of EEG signals. The brain signals obtained are mixed with artifacts at integrated level. The Independent Component Analysis samples the peaks of signals mixed with artifacts and produce segmented outputs. The Discrete Wavelet Transform divides the signal in a multi-level platform until the reception of significant result. The tests were performed on an outsourced EEG data. The Double Density Algorithm was applied in this paper and the comparison was made with DWT. The performance evaluation is measured by PSNR and MSE and finally the comparative analysis of two Data sets is presented in a tabular manner. Hence it is obtained that the method presented was a step superior of the other artifact removal methods.

\section{References}

[1] http://cognitrn.psych.indiana.edu/busey/ eegsemi nar/pdfs/EEGPrimer Ch6.pdf

[2] Iván Manuel Benito Núñez, "EEG Artifact Detection”. Department of Cybernetics Czech Technical University, Prague.

[3] Jorge Baztarrica Ochoa, "EEG Signal Classification for Brain Computer Interface Applications". http://www.texnogen.org/ wvt/bz.pdf

[4] Tzyy-Ping Jung \& Scott Makeig, "Removing Artifacts from EEG” http://sccn.ucsd.edu/ jung/ artifact.html

[5] Arnaud Delorme, Terrence Sejnowski, and Scott Makeig, "Enhanced detection of artifacts in EEG data using higher-order statistics and independent component analysis" Elsevier, NeuroImage 34 (2007) 14431449.

[6] P. Senthil Kumar, R. Arumuganathan, K. Sivakumar, and C. Vimal, "Removal of Ocular Artifacts in the EEG through Wavelet Transform without using an EOG Reference Channel” Int. J. Open Problems Compt. Math., Vol. 1, No. 3, December 2008.

[7] Joseph T. Gwin, Klaus Gramann, Scott Makeig, and Daniel P. Ferris, "Removal of Movement Artifact From High-Density EEG Recorded During Walking and Running” J Neurophysiol 103: 3526-3534, 2010.

[8] M. Ungureanu, C. Bigan, R. Strungaru, V. Lazarescu, "Independent Component Analysis Applied in Biomedical Signal Processing”. Measurement Science Review, Volume 4, Section 2, 2004.

[9] W. Selesnick, "The double-density dual-tree DWT," IEEE Trans. Signal Processing, vol. 52, no.5, 2004, pp. 1304-1314. 
[10] Ivan W. Selesnick, "The Double Density Dwt" Polytechnic University. Brooklyn, NY.

[11]"Rodrigo Quian Quiroga, EEG, ERP and single cell recordings database". http://www.vis.caltech.edu/ $\sim$ rodri/data.htm

[12] Ivan W. Selesnick, "The Double-Density Dual-Tree DWT" IEEE TRANSACTIONS ON SIGNAL PROCESSING, VOL. 52, NO. 5, MAY 2004.

[13]Flexer A., Bawer H., Pripfl J., Dorffner G.:Using ICA for removal of ocular artifacts in EEG recorded from blind subjects :, Neural Networks 18.7 , 2005, 998-1005.

[14] Klados and Manousos A.: REG-ICA: A hybrid methodology combining Blind Source Separation and regression techniques for the rejection of ocular artifacts: Biomedical Signal Processing and Control 6.3, 2011, 291-300.

[15] Lindsen, Job P., and Joydeep B.: Correction of blink artifacts using independent component analysis and empirical mode decomposition: Psychophysiology 47.5, 2010, 955-960.

[16] Escudero J., Hornero R., Abasolo D., Fernandez A.: Quantitative evaluation of artifact removal in real magnetoencephalogram signals with blind source separation: Annals of biomedical engineering 39.8, 2011, 2274-2286.

[17]Flexer A., Bauer H, Pripfl J, Dorffner G.:Using ICA for removal of ocular artifacts in EEG recorded from blind subjects: Neural Networks 2005;18,998-1005.

[18] Hyv"arinen A., Pajunen P.: Nonlinear independent component analysis: existence and uniqueness results: Neural Networks 1999, 12, 209-219.

[19] Chao, Jih-Cheng, and Scott C. Douglas. : A robust complex FastICA algorithm using the huber Mestimator cost function; Independent Component Analysis and Signal Separation: Springer Berlin Heidelberg, 2007, 152-160.

[20] Arora S., Ge R., Moitra A., Sachdeva S.: Provable ICA with unknown Gaussian noise, and implications for Gaussian mixtures and autoencoders : arXiv preprint arXiv:1206.5349, 2012.

\section{Author'S Introduction}

Dr. Shailja Shukla received B.E. degree in Electrical Engg. from Jabalpur Engg. College, Jabalpur in 1984 and the Ph.D. degree in Control System from Rajiv Gandhi Technical University, Bhopal in 2002. She is currently Professor in Electrical Engg. and the Chairperson of the Department of Computer Science and Engg. At Jabalpur Engg. College, Jabalpur. Her research interest on Large Scale Control Systems, Soft Computing and include Machine Learning, Face Recognition, image processing and Digital Signal Processing. She has been the Organizing Secretary of International Conference on Soft Computing and Intelligent Systems. She has published more than 60 Research papers in International/National Journals and conferences. She is Editorial member of many International Journals.

Vandana Roy received B.E. degree in Electronics and communication Engg. from HCET, Jabalpur in 2001 and the M. Tech. degree in Digital Communication from Rajiv Gandhi Technical University, Bhopal in 2010 . She is currently Professor in GGITS Jabalpur in Electronic Engg.. Her research interest are Communication, Image processing and Digital Signal Processing. She has published more than 14 Research papers in International/National Journals and conferences.

How to cite this paper: Vandana Roy, Shailja Shukla,"Artifacts Removal of EEG Signals By the Application of ICA and Double Density DWT Algorithm", IJEM, vol.4, no.2, pp.42-55, 2014.DOI: 10.5815/ijem.2014.02.04 